slightly lower than target in IDA (43\%) and suspected CD $(40 \%)$ indications, reflecting that better selection of cases can increase CE diagnostic yield. A standardized approach to audit $\mathrm{CE}$ is necessary and should follow KPI as a standard for comparison.

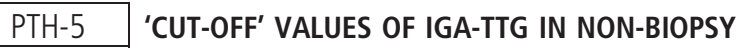 DIAGNOSTIC PATHWAYS FOR COELIAC DISEASE NEED TO BE ASSAY-SPECIFIC}

Richard Vaughan*, Francesca Melindo, Benjamin Corney, Hannah Williams, Alice Bone, James Berrill. Royal Glamorgan Hospital, Llantrisant, UK

\subsection{6/gutjpl-2021-BSG.284}

Introduction The British Society of Gastroenterology (BSG) interim guidelines (June 2020) on making a non-biopsy diagnosis of coeliac disease included an IgA anti-tissue transglutaminase (TTG) level $\geq 10 \times$ upper limit of normal (ULN) in the pathway. However this was only recommended for an ELISA-based assay. It advised that local TTG assay reliability needs correlating to pathology as part of any local audit.

Since June 2019, our health board switched from an ELISA based assay to a chemiluminescence based assay for TTG measurement. This study aims to compare the two distinct assays, and propose an appropriate 'cut-off' level for the chemiluminescence assay.

Method In this retrospective, observational study across two sites, all elevated TTG results in adult patients from January 2018 to December 2019 were identified from biochemistry records. Patients already known to have coeliac disease were excluded from analysis. In patients with multiple elevated TTG results only the earliest result was included. Data was collected on patient demographics, waiting times, and duodenal biopsy results. Prior to June 2019 an ELISA based assay was used with TTG results $<10 \mathrm{U} / \mathrm{ml}$ considered negative. Since June 2019 a chemiluminescence assay (Bioflash) was employed, with TTG results $<20 \mathrm{U} / \mathrm{ml}$ reported as negative.

Results In total, 192 elevated TTG results were included in the analysis. One hundred and nine patients had duodenal biopsies performed, 73 patients were not referred or declined investigation, and 10 patients were still waiting for duodenal biopsy at the time of analysis in May 2021. The median waiting time for these 10 patients still awaiting biopsy was 456 days.

Using the ELISA based assay, $96 \%$ patients $(n=27)$ with TTG $>10 \times$ ULN had positive duodenal biopsies, whereas positive biopsies were only present in $56 \%$ patients $(n=9)$ with TTG between $5-10$ x ULN.

Using the Chemiluminescence assay, 90\% patients $(n=21)$ with TTG $>10 \times$ ULN had positive duodenal biopsies, however positive biopsies were also present in $89 \%$ patients $(n=9)$ with TTG between 5 - 10 x ULN.

Discussion These results support the BSG guidance for nonbiopsy diagnosis of coeliac disease using a pathway that includes TTG $>10 \mathrm{x}$ ULN measured by an ELISA assay. However for the chemiluminescence assay (Bioflash) it appears that a TTG level $\geq 5 \mathrm{x}$ ULN may be a more appropriate 'cut-off' level to use in the pathway. Further work is required to confirm this as the numbers in this study are small. This study also shows the long time periods that patients in some centres are waiting for their duodenal biopsies, likely exacerbated by the COVID pandemic, and thus the importance of establishing a non-biopsy protocol where appropriate.

\section{PTH-6 UTILITY OF A HOME HYDROGEN BREATH TESTING PATHWAY FOR DIAGNOSIS OF SMALL INTESTINAL BACTERIAL OVERGROWTH}

Jabed Ahmed*, Pritpal Padam, Aruchuna Ruban. Imperial Healthcare NHS Trust, London, UK

\subsection{6/gutjnl-2021-BSG.285}

Introduction Small Intestinal bacterial overgrowth (SIBO) can lead to persistent diarrhoea, bloating and abdominal pain in patients. Hydrogen Breath testing (HBT) is the first line investigation but since the COVID-19 pandemic its utility has been limited as it is considered an aerosol generating procedure. We piloted a new testing pathway at a London teaching hospital in which HBT kits were sent out for patients to perform the test at home and sent back for analysis.

Aims \& Methods Patients on our HBT waiting list between April 2019 to November 2020 were contacted and assessed for symptomology, validity for home testing and consent for participation. 39 tests were mailed out to patients and data was collected on the response rate, number of positive results, alongside a patient satisfaction survey.

Results A total of 108 patients (M 42: F 66) were identified to be on the waiting list for a hydrogen breath test. 8 patients were removed due to symptom improvement, being discharged from clinic or due to cancellation of request. All 100 patients were contacted with 91 responding. 88 (96.7\%) agreed to undergo the home testing for SIBO. 86.4\% (76/88) reported to still be symptomatic with $13.6 \%(12 / 88)$ reporting no longer having symptoms.

39 home testing kits were sent and 14 (36\%) kits were returned over a 2 month period.

$10(26 \%)$ were hydrogen breath test positive. (Figure 1).

The average score given for ease of use for the home kit was $3.7 / 5.10 / 14(71 \%)$ of the patients accessed the video link and $4.3 / 5$ said they would recommend this testing method to others.

A follow up call was undertaken to ascertain the poor response rate from patients and common feedback included difficulty in understanding instructions, restrictive diet required prior to testing and time constraints.

Conclusions The initial results from our pilot study show promise and suggest that home testing is a realistic, viable and cost-effective first line option for the management of SIBO in the post covid era.

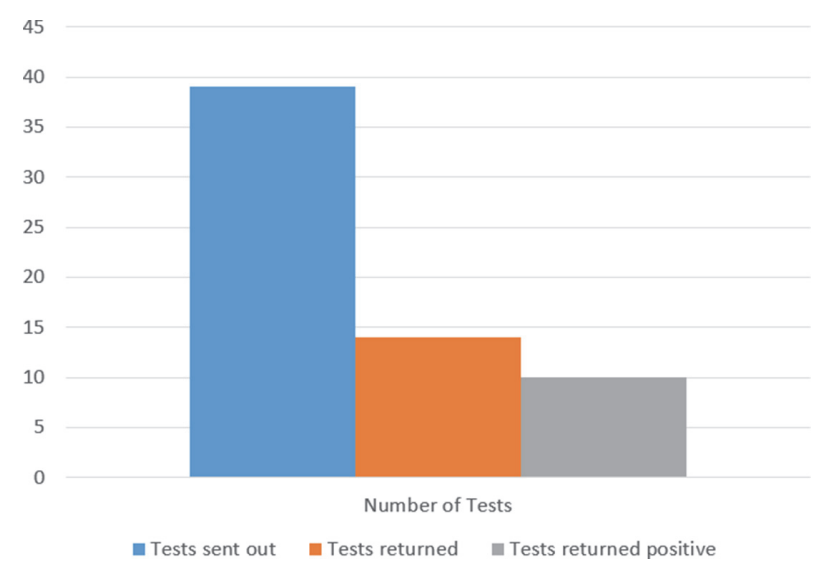

Abstract PTH-6 Frigure 1 Home breath tests 
The poor response rate is a concern and steps to remedy this may include follow up telephone consultations, patient education and encouraging patients to access the video link to assist them in performing the test.

\section{Nutrition}

\section{PTH-7 REVIEW OF PATIENTS WITH COELIAC DISEASE IN NORTHERN ENGLAND}

${ }^{1}$ Mohammed Adnan Khan*, ${ }^{2} E$ Sainsbury, ${ }^{3} E$ Johns, ${ }^{4} D$ Hobday, ${ }^{5} L$ Walker, ${ }^{6}$ Ross, ${ }^{7}$ David Oliver, ${ }^{8}$ Chris Wells, ${ }^{1} \mathrm{D}$ Smith, ${ }^{1} \mathrm{NP}$ Thompson. ${ }^{1}$ Freeman Hospital, Newcastle, Newcastle, UK; ${ }^{2}$ South tyneside hospital; ${ }^{3} Q E$ hospital, Gateshead; ${ }^{4}$ Sunderland royal hospital, Sunderland; ${ }^{5}$ University hospital of Durham, Durham; ${ }^{6}$ Cumberland Infirmary, Carlisle; ${ }^{7}$ James cook university hospital, Middlesbrough; ${ }^{8}$ North Tees hospital, Stockton on tees, UK

\subsection{6/gutjnl-2021-BSG.286}

Introduction The BSG and NICE recommend long-term regular follow-up of patients with Coeliac disease (CD). It is unclear when and what to be done when following a newly diagnosed CD patient and who should perform this review. The Northern Nutrition Network is multi-disciplinary, meets regularly and involves all acute Trusts in the north-east and north Cumbria. It is recommended that we check FBC, U\&Es, LFTs, bone profile, Vit D and CD serology.

Aim To assess the follow up of patients with CD across the region.

Method A retrospective review of CD follow up using patient's records. All regional acute Trusts were invited to participate. A questionnaire was developed and circulated. Follow up data, for upto 2 years after the diagnosis of CD, were recorded for up to 20 patients per Trust, diagnosed after 1.1.2017.

Results 141 responses received from 8 of 9 trusts. Median age was 41 years. 38\% (54/141) were male. Histology was consistent with $\mathrm{CD}$ in all patients. EMA was positive when tested in 92\%, negative in 8\% - not tested 17\%. TTG was positive when tested in 95\%, negative in 5\% - not tested in 6\%. TTG values varied from $6.4 \mathrm{U} / \mathrm{ml}$ to $>1000 \mathrm{U} / \mathrm{ml}$.

Following diagnosis, $70 \%$ of $\mathrm{CD}$ patients were followed up by gastroenterologists in first 4 months, $88 \%$ in their first year and $89 \%$ in 2 years. Only $11 \%(16 / 141)$ did not see a gastroenterologist in the first 24 months after diagnosis. 90\% of $\mathrm{CD}$ patients were seen by dietician in first 4 months, 91\% in first year and $91 \%$ in 2 years following diagnosis. 9\% were not seen by dietician. There were 2 trusts in which patients were followed up by nurse specialist as well.

Weight was measured at review in $92 \%$ in 1 st year and $71 \%$ in 2 nd year. BMI was recorded for $71 \%$. Dietary compliance was recorded in $85 \%$ at review: $60 \%$ were fully compliant to gluten free diet (GFD), 15\% had occasional lapses, only $10 \%$ were not compliant.

$4 \%$ did not have FBC or LFTs and 2\% U\&Es checked in first 24 months. 12\% did not have calcium and 41\% did not have Vit D checked in first 24 months. Serology was checked in $83 \%$ in 1 st year, $53 \%$ in the 2 nd year, $12 \%$ did not have serology in first 24 months. Following review of blood tests results, actions taken on abnormal results: Folic acid supplemented 6 times, Vit D supplemented 17 times, Iron 15 times, Vit B12 4 times and Calcium 3 times.
Repeat duodenal biopsy was undertaken within 2 years in $15 \%$ of patients. Estimation of fracture risk was recorded in $16 \%$ but BMD measurement within 2 year was in $66 \%$. In $18 \%$ advice was given on vaccination during the review.

Conclusion Practice in the follow-up of patients with CD varied. Blood tests are checked regularly but we are poor in recording vaccination advice and perform BMD rather than estimate fracture risk non-invasively. Repeat duodenal biopsy is unusual. There is a need of robust follow up regimens across the region.

\section{PTH-8 NOMOGRAM RELIABILITY FOR PREDICTING SURVIVAL IN PATIENTS WITH INCURABLE CANCER REFERRED FOR HOME PARENTERAL NUTRITION}

${ }^{1,2}$ Colette Kirk*, ${ }^{1}$ Hayley Leyland, ${ }^{1}$ Nick Thompson, ${ }^{1}$ Christopher Mountford ${ }^{1}$ Courtney Hankins, ${ }^{3}$ Hannah Cook, ${ }^{1}$ Jessica McDonald, ${ }^{1}$ Lisa Gemmell. ${ }^{1}$ The Newcastle upon Tyne Hospitals NHS Foundation Trust, Newcastle upon Tyne, UK; ${ }^{2}$ NIHR Newcastle Biomedical Research Centre, Newcastle upon Tyne, UK; ${ }^{3}$ Northumbria Healthcare NHS Foundation Trust, Newcastle upon Tyne, UK

\subsection{6/gutjnl-2021-BSG.287}

Introduction In the presence of bowel obstruction, enterocutaneous fistula, short bowel, or severe mucosal disease, patients with incurable cancer are increasingly being referred for consideration of home parenteral nutrition (HPN). The decision to commence such treatment largely relies on expert opinion rather than robust data showing survival outcome. To address this shortcoming, a nomogram to predict median survival length in palliative cancer patients treated with HPN has been developed and validated. ${ }^{1}$ The nomogram is based on Glasgow prognostic score (CRP \& albumin), primary cancer, metastases and Karnofsky performance status. The aim of this study was to assess the reliability and clinical value of the nomogram.

Methods The nomogram was applied ambidirectionally to adult patients referred for palliative HPN between $1 / 3 / 15$ and $7 / 7 / 20$ at one tertiary HPN centre. Patients receiving chemotherapy or radiotherapy at the point of referral or during HPN treatment, and patients with neuroendocrine tumours were excluded. Intraclass correlation coefficient (ICC) was used to measure the reliability of the nomogram.

Results 35 patients were identified. Eight patients were excluded due to commencing chemotherapy. Of the 27 remaining patients, $15(66 \%)$ were female. 16 (59\%) patients had primary GI cancers, six (22\%) ovarian, and five (19\%) other forms of cancer. Overall mean survival was 114 days (22-433) versus 104 days (30-200) for predicted survival $(p=0.746)$. The nomogram over predicted survival in $59 \%$ of cases and under predicted in 33\%. The predictions for seven patients (26\%) were within 20\% of their actual survival, 12 patients (44\%) were within 50\%, and the remaining patients between 50 and $248 \%$. The ICC was 0.327 with a confident interval of -0.64-0.627, indicative of poor reliability. ${ }^{2}$

Conclusion Although the $p$ value suggests no significant difference between predicted and actual survival length, our study is limited by the small sample size. We considered a $20 \%$ variance between predicted and actual survival clinically acceptable; only a quarter of patients were within this range. Our study therefore does not support the use of the nomogram to predict survival in patients referred for palliative HPN and we should continue to use clinical acumen when considering such 